BULL. AUSTRAL. MATH. SOC.

\title{
DEGREES GIVING INDEPENDENT EDGES \\ IN A HYPERGRAPH
}

\author{
David E. Daykin and Roland Häggkvist
}

For r-partite and for r-uniform hypergraphs bounds are given

for the minimum degree which ensures $d$ independent edges.

\section{Introduction and statement of results}

(i) HYPERGRAPHS

Let $c, r, s$ be positive integers with $2 \leq r$ and let $S=\{1,2, \ldots, s\}$. A set $H$ of subsets of $S$ is a hypergraph. The members of $H$ are called edges. Two edges $\alpha, \beta \in H$ are independent if $\alpha \cap \beta=\emptyset$. The degree $\operatorname{deg}_{H}(x)$ of $x \in S$ in $H$ is the number of members of $H$ containing $x$. We write $\delta(H)$ for $\min \left\{\operatorname{deg}_{H}(x)\right\}$ over $x \in S$. Let $B$ be the set of all $\alpha \subset S$ of cardinality $|\alpha|=r$. In this paper each $H \subset B$ so $H$. is an $r$-graph or $r$-uniform hypergraph. We are concerned with the least number $\omega$ such that every $H$ with $\omega<\delta(H)$ has more than $d$ independent edges. Related problems are dealt with in the references.

(ii) $r$-PARTITE $r$-GRAPHS

Suppose $S$ is a disjoint union $S=R_{1} \cup \ldots \cup R_{r}$ with $\left|R_{i}\right|=c$ for $1 \leq i \leq r$ so $s=c r$. Let $A$ be the set of all $\alpha \subset S$ such that $\left|\alpha \cap R_{i}\right|=1$ for $1 \leq i \leq r$. In this case any $H \subset A \subset B$ is an $r$-partite hypergraph.

Received 14 August 1980. 
THEOREM 1. If $0 \leq d<c$ and $H$ is r-partite as above with

$$
\delta(H)>\left\{e^{r-1}-(c-d)^{r-1}\right\}(r-1) / r
$$

then $H$ has more than $d$ independent edges.

To see how close this theorem gets to $\omega$ consider

EXAMPLE 1. Put $d=q r+p$ with $0 \leq p<r$. For $1 \leq i \leq p$ select $q+1$ elements of $R_{i}$. For $p<i \leq r$ select $q$ elements of $R_{i}$. Let $H$ consist of all $\alpha \in A$ which contain at least one of the $d$ selected elements. Then $\delta(H)$ is approximately $c^{r-1}-\left(c-r^{-1} d\right)^{r-1}$ but $H$ does not have $d+1$ independent edges.

(iii) GENERAL $r$-GRAPHS

EXAMPLE 2. Select $d$ elements of $S$ and let $H$ consist of all $\alpha \in B$ which contain at least one of the selected elements. Then $\delta(H)=\left(\begin{array}{l}s-1 \\ r-1\end{array}\right)-\left(\begin{array}{c}s-d-1 \\ r-1\end{array}\right)$ but $H$ does not have $d+1$ independent edges.

THEOREM 2 (Bollobas, Daykin and Erdös). If $0 \leq d$ and $2 r^{3}(d+2)<s$ and

$$
\delta(H)>\left(\begin{array}{l}
s-1 \\
r-1
\end{array}\right)-\left(\begin{array}{c}
s-d-1 \\
r-1
\end{array}\right)
$$

then $H$ has more than $d$ independent edges.

That this theorem has evaluated $\omega$ is shown by Example 2. It appears in [1] where it is in fact proved that all $H$ with a fixed number of independent edges and high $\delta(H)$ are subhypergraphs of Example 2. In Theorem 2 it is required that $s$ be large. Without this requirement we bound $\omega$ in

THEOREM 3. If $r$ divides $s$ and

$$
\delta(H)>\left\{\left(\begin{array}{l}
s-1 \\
r-1
\end{array}\right)-\left(\begin{array}{c}
s-d r-1 \\
r-1
\end{array}\right)\right\}(r-1) / r
$$

then $H$ has more than $d$ independent edges.

For Theorems 1 and 3 we prove slightly more than what is stated. Namely that if $C_{1}, \ldots, C_{d}$ is any maximum set of independent edges, and if $E$ is any possible edge in $S \backslash\left\{C_{1} \cup \ldots \cup C_{d}\right\}$ then $E$ has low average 
degree. We believe the condition $r$ divides $s$ can be removed but were not able to do so.

\section{Proof of Theorem 1}

Part (i). Assume that $1 \leq d<c$ and $H$ has $d$ independent edges $c_{1}, \ldots, c_{d}$ but not $d+1$. Choose arbitrarily members $c_{d+1}, \ldots, c_{c}$ of $A$ so that $S$ is the disjoint union $S=C_{1} \cup \ldots \cup C_{c}$. We label the elements $x(i, j)$ of $S$ so that

$$
\begin{aligned}
& c_{j}=\{x(1, j), \ldots, x(r, j)\} \text { for } 1 \leq j \leq c, \\
& R_{i}=\{x(i, 1), \ldots, x(i, c)\} \text { for } 1 \leq i \leq r .
\end{aligned}
$$

The reader will probably find it helpful to think of $S$ as the elements of a matrix. Then $c, C$ refer to columns and $r, R$ to rows. We write $D$ for the union of the $d$ independent edges $D=C_{1} \cup \ldots \cup C_{d}$ and $E$ for $c_{c}$ the end column in the matrix.

We will use the cyclic permutation $\sigma$ on $n$ distinct positive integers $w_{1}, \ldots, w_{n}$ defined by $\sigma w_{n}=w_{1}$ and $\sigma w_{i}=w_{i+1}$ otherwise. We proceed to partition $A$.

Part (ii). Given $\alpha=\left\{x\left(1, j_{1}\right), \ldots, x\left(r, j_{r}\right)\right\} \in A$ let $\left\{w_{1}, \ldots, w_{n}\right\}=\left\{j_{1}, \ldots, j_{p}\right\}$ with $1 \leq w_{1}<\ldots<w_{n} \leq c$. Note that $n \leq r$. Then put.

$$
K(\alpha)=\left\{\left\{x\left(1, \sigma^{e} j_{1}\right), \ldots, x\left(r, \sigma^{e} j_{r}\right)\right\}: 1 \leq e \leq n\right\} .
$$

We say that the members of $K(\alpha)$ are obtained by rotating $\alpha$. The sets $K(\alpha)$ are the equivalence classes of our partition of $A$.

Part (iii). Let $X=\{\alpha: \alpha \in A, \alpha \cap D \neq \emptyset\}$. Then by definition of $d$ we have $H \subset X$. Let $K$ be the set of equivalence classes in the partition of $A$. If $K \in K$ then either $K \subset X$ or $K \cap X=\varnothing$. For $L \subset A$ define

$$
\Delta(L)=\sum(x \in E) \operatorname{deg}_{L}(x)
$$

Let $Y=\{\alpha: \alpha \in A, \alpha \cap E \neq \emptyset\}$. If $K \in K$ then either $K \cap Y=\emptyset$ or 
$K \subset Y$ according as $0=\Delta(K)$ or not. For all $L \subset A$ we have $\Delta(L)=\Delta(L \cap Y)$ and in particular $\Delta(H)=\Delta(H \cap X \cap Y)$.

Assume for the moment that

$$
r \Delta(H \cap K) \leq(r-1) \Delta(K) \text { for all } K \in K \text { with } K \subset X \cap Y .
$$

Then we have

$$
r \Delta(H)=r \sum \Delta(H \cap K) \leq(r-1) \sum \Delta(K)=(r-1) \Delta(X \cap Y),
$$

where summation is over $K \in K$ with $K \subset X \cap Y$.

Part (iv). Clearly $\Delta(A)=r c^{r-1}$ and $\Delta(X \cap Y)=r\left(c^{r-1}-(c-d)^{r-1}\right)$. So the result follows by (5). It remains to prove (4).

Part (v). Suppose $K \in K$ and $K \subset X \cap Y$. If $\alpha \in K$ then the other members of $K$ are obtained by rotating $\alpha$. Hence every $x \in E$ is in exactly one member of $K$ and so $\Delta(K)=r$. If $k=|K|$ then $K$ consists of $k$ independent members of $A$. Again by the rotation $K \cap C_{j} \neq \varnothing$ for less than $k$ of the $j$ in $1 \leq j \leq d$. Therefore if $K \subset H$ we could remove these $c_{j}$ from $C_{1}, \ldots, c_{d}$ and adjoin $K$ to get more than $d$ independent edges of $H$. Hence $K \notin H$ and so $\Delta(H \cap K) \leq r-1$ and this proves (4).

\section{Proof of Theorem 3}

We use ideas from the last proof. In fact we have chosen our notation so that parts of the last proof carry over unchanged, provided $A$ now means the set $B$ of all $\alpha \subset S$ with $|\alpha|=r$. Do not be deceived. Although the writing is the same the meaning is different.

Part (i). As before. Note that before the $R^{\prime} s$ were given but now they are defined by (2).

Part (ii). Given a row vector $v=(v(1), \ldots, v(c))$ of non-negative integers $v(j)$ let

$$
W=\left\{w_{1}, \ldots, w_{n}\right\}=\{j: 1 \leq j \leq c \text { and } 0<v(j)\},
$$

with $1 \leq w_{1}<\ldots<w_{n}$. Note that $n \leq c$. Now define a permutation $\pi$ of $\{1, \ldots, c\}$ by $\pi j=\sigma j$ if $j \in W$ but $\pi j=j$ otherwise. Finally 
put

$$
V=V(v)=\left\{\left(v\left(\pi^{e} 1\right), \ldots, v\left(\pi^{e} c\right)\right): 1 \leq e \leq n\right\} \text {. }
$$

For example if $v=(1,0,2,1,0,0,2)$ then $n=4$ and $W=\{1,3,4,7\}$ and $V$ is $v$ and $(2,0,1,2,0,0,1)$.

Given $\alpha \in A$ put $v(j)=\left|\alpha \cap c_{j}\right|$ for $I \leq j \leq c$. In this way $\alpha$ yields a row vector $v$. In turn $v$ yields a set $V$ of row vectors as above. We use $V=V(\alpha)$ to define $K \subset A$ by

$$
K=K(\alpha)=\{\beta: \beta \in A \text {, row vector of } \beta \in V(\alpha)\} \text {. }
$$

Clearly the set $K$ of all sets $K(\alpha)$ over $\alpha \in A$ are the equivalence classes of a partition of $A$.

Part (iii). As before.

Part (iv). Clearly $\Delta(A)=r\left(\begin{array}{l}s-1 \\ r-1\end{array}\right)$ and $\Delta(X \cap \cap \quad Y)=r\left\{\left(\begin{array}{l}s-1 \\ r-1\end{array}\right)-\left(\begin{array}{c}s-d r-1 \\ r-1\end{array}\right)\right\}$. So the result follows by (5). It remains to prove (4).

Part (v). Choose any $K \in K$ with $K \subset X \cap Y$ and fix it. An ordering of $C_{j}$ is a bijection $\lambda_{j}: C_{j} \rightarrow\{1,2, \ldots, r\}$ and the number of these is $r !$. For $1 \leq j \leq c$ let $\lambda_{j}$ be an ordering of $c_{j}$. We say that $\alpha \in K$ is good in $\lambda=\left(\lambda_{1}, \ldots, \lambda_{c}\right)$ if

$$
\underset{1 \leq j \leq c}{U}\left\{\underset{x \in \alpha \cap C_{j}}{U} \lambda_{j}(x)\right\}=\{1,2, \ldots, r\} .
$$

If we think of $\lambda$ as reordering the columns of $S$ as a matrix then $\alpha$ is good in $\lambda$ if it has exactly one element in each row of the reordered $S$.

If $\alpha, \beta \in K$ then the numbers $\left|\alpha \cap C_{j}\right|$ are the same as the numbers $\left|\beta \cap C_{j}\right|$ in some order. Hence $\alpha$ and $\beta$ are good in the same number $t$ of the $\lambda$. For each $\lambda$ let $F(\lambda)$ and $G(\lambda)$ be the set of all $\alpha$ in $K$ and $H \cap K$ respectively which are good in $\lambda$. Then

$$
\Delta(H \cap K)=t \sum \Delta(G(\lambda)) \text { and } \Delta(K)=t \sum \Delta(F(\lambda)) \text {, }
$$

where summation is over $\lambda$. Assume for the moment that

$$
r \Delta(G(\lambda)) \leq(r-1) \Delta(F(\lambda)) \text { for all } \lambda \text {. }
$$


Then (4) follows immediately using (6).

Part (vi). Choose any $\lambda$ and fix it. For simplicity write $F, G$ instead of $F(\lambda), G(\lambda)$. After $S$ has been reordered by $\lambda$ we renumber the elements $x(i, j)$ of $S$ so that (I) and (2) again hold. Given any $\alpha \in F$ we define the set $K(\alpha)$ exactly as in (3). To avoid confusion let $K(\alpha)$ be called $J$. Because the members of $J$ are obtained by rotating $\alpha$ they are all in $K$. Also by construction they are all good in $\lambda$. In fact the various $J$ partition $F$. Exactly as in Part (v) of the proof of the last theorem we find that $\Delta(J)=r$ and $\Delta(H \cap J) \leq r-1$. Hence

$$
r \Delta(G)=r \sum \Delta(H \cap J) \leq r \sum(r-1)=(r-1) \sum r=(r-1) \sum \Delta(J)=(r-1) \Delta(F),
$$

where summation is over the equivalence classes $J$ which partition $F$, and this proves ( 7 ).

\section{References}

[1] B. Bollobss, D.E. Daykin and P. Erdös, "Sets of independent edges in a hypergraph", Quart. J. Math. Oxford Ser. (2) 27 (1976), 25-32.

[2] Alan Brace, "Some combinatorial cover theorems" ( $\mathrm{PhD}$ thesis, University of Western Australia, Nedlands, 1971).

[3] Alan Brace and D.E. Daykin, "A finite set covering theorem", Bulz. Austral. Math. Soc. 5 (1971), 197-202.

[4] Alan Brace and D.E. Daykin, "A finite set covering theorem II", BulZ. Austral. Math. Soc. 6 (1972), 19-24.

[5] Alan Brace and D.E. Daykin, "A finite set covering theorem III", BulZ. Austraz. Math. Soc. 6 (1972), 417-433.

[6] A. Brace, D.E. Daykin, "A finite set covering theorem. IV", Infinite and finite sets, Vol. I, 199-203 (Colloq., Keszthely, 1973. Colloq. Math. Soc. János Bolyai, 10. North-Holland, Amsterdam, 1975).

[7] Alan Brace, D.E. Daykin, "Sperner type theorems for finite sets", Combinatorics, 18-37 (Proc. Conf. Combinatorial Math., Math. Inst., Oxford, 1972. Inst. Math. Appl., Southend-on-Sea, 1972). 
[8] D.E. Daykin, "Minimum subcover of a cover of a finite set", Problem E2654, Amer. Math. Monthly 85 (1978), 766.

Department of Mathematics,

University of Reading,

Whiteknights,

Reading,

Berkshire RG6 2AX,

England;

Institut Mittag-Leffler,

Auravägen 17,

S-182 62 Djursholm,

Sweden. 